\title{
Application of Virtual Experiment in Maker Education
}

\author{
Kexin Yin*, Nan Wang \\ Institute of Educational Technology, Beijing University of Posts and Telecommunications, Beijing, China
}

\section{Email address:}

15169028665@163.com (Kexin Yin), etwangnan@126.com (Nan Wang)

*Corresponding author

\section{To cite this article:}

Kexin Yin, Nan Wang. Application of Virtual Experiment in Maker Education. Science Discovery. Vol. 6, No. 5, 2018 , pp. $392-398$. doi: 10.11648/j.sd.20180605.23

Received: September 14, 2018; Accepted: October 15, 2018; Published: October 18, 2018

\begin{abstract}
In 2016, Maker became a hot spot in the field of educational technology. Countries around the world has paid special attention to the development of makers and innovations, and Maker Education also developed rapidly with the continuous advancement of cutting-edge technology and the renewal of people's educational concepts. The theoretical researches and applications related to it are also increasing. Virtual experimental technology has developed stably in recent years and has been widely used in all aspects of education and teaching. The application of virtual experiment technology in the field of Maker Education can not only solve the existing problems of Maker Education, but also promote the development of Maker Education in the future. This paper analyzes the related literatures and cases of virtual experiment technology and the development of maker education in recent years. Taking the application of virtual experiment in maker education as the starting point, the existing problems of maker education, the application status of virtual experiment in maker education as well as analysis of application principles and application examples are analyzed, finally the paper puts forward their own ideas for the application prospects of virtual experiments in the field of maker education. Therefore, the researches in this paper can provide a useful reference for the future development model of Maker Education.
\end{abstract}

Keywords: Maker Education, Virtual Experiment Technology, Virtual Simulation Teaching

\section{虚拟实验在创客教育中的应用探索}

\section{殷可欣”，王楠}

教育技术研究所, 北京邮电大学, 北京市, 中国

\section{邮箱}

15169028665@163.com（殷可欣）, etwangnan@126.com（王楠）

摘要：创客在2016年成为教育技术研究的热点, 世界各国对于创客、创新的发展尤为重视, 而创客教育也随着前沿技 术的不断进步和人们教育理念的更新而迅速发展成熟,与其相关的理论研究及应用也日趋增多。虚拟实验技术近几年来 发展稳定并且广泛应用于教育教学的各个环节。而将虚拟试验技术应用于创客教育, 不仅能解决创客教育现存的问题, 而且对创客教育未来的发展有着很好的促进作用。本文研究分析了近年来虚拟实验技术及创客教育发展的相关文献资 料和案例, 以虚拟实验在创客教育中的应用作为出发点, 从创客教育现存的问题、虚拟实验在创客教育中的应用现状、 应用原则以及应用实例等方面进行分析, 最后提出自己对于虚拟实验的未来应用在创客教育领域的想法。因此, 本文 的研究可对创客教育的未来发展模式提供有益参考。

关键词: 创客教育, 虚拟实验技术, 虚拟仿真教学 


\section{1. 引言}

科学技术是第一生产力。而科学技术的关键在于创 新。21世纪, 教育的本质已经由知识的积累转向创意创新 [1]。近年来, 随着世界范围内的创新、创业和智创高潮的 到来, “创客”、“创客教育”、“创客空间”等词汇进入大众 视野, 不管是美国、欧洲国家还是中国等亚洲各国, 对创 客、创新的重视程度不断增加。2016年6月18日, 在第三 届白宫“创客嘉年华”上美国政府宣布: “创客国家计划”已 经调动政府、企业、教育和社会各方面的力量支持创客运 动和创客教育的发展。2016年, 中华人民共和国教育部在 关于印发《教育信息化“十三五”规划》和《国家创新驱动 发展战略纲要》文件的通知中指出, 支持学校开展创客教 育方面实践[2]。目前, 无论是高校还是各地中小学都在大 力开展创客教育, 社会上也出现很多创客公司, 创客教育 也随着前沿技术的不断进步和人们教育理念的更新而迅 速发展成熟。创客教育的出现势必会对当前主流的传统教 育产生强烈的冲击, 但这也是新时代教育发展的时代需 求。

而创客空间作为创客教育的主要承载形式, 是创客聚 集、交流和协作创造的场所, 也是学生培养创新能力、实 践能力、团队合作能力的聚集地。因此创客空间必须具有 开放性、灵活性、动态性、交互性等特点。虽然目前世界 各国有很多的创客空间, 很多高校、中小学也在进行创客 空间的建设，但更多的集中于实体创客空间的实现。创客 教育要想更大范围的得到普及和推广, 虚拟空间的建设必 不可少，而虚拟实验技术恰好可以满足这一需求。

\section{2. 绪论}

\section{1. 研究背景}

\subsection{1. 时代背景}

在 2010 年 5 月中华人民共和国国务院常务会议中审议 通过的《国家中长期教育改革和发展规划纲要 (20102020 年) 》中指出, 目前我国面临的主要问题是创新型 人才的贵乏, 在未来 10 年, 我国将创新型人才的培养作为 教育改革的重点。[3]而传统教育经过长时间的发展逐渐暴 露出其自身的弊端, 即着重知识传授轻能力培养、重智力 因素轻非智力因素、重课业轻创造[4]。因此, 改革现有的 教育模式及教育观念, 培养适应时代发展的创新型人才是 当前亟待解决的问题。而创客教育应运而生。将创客教育 引入学校教育, 对培养学生的综合运用能力、创新实践能 力有着至关重要的作用。

\subsection{2. 创客教育的发展需要}

近年来, 创客教育席卷全球。创客教育的教学模式以及 本身具有的创新观念得到越来越多的关注和认可, 许多高 校、中小学甚至社会上的企业机构都纷纷启动了创客教育相 关的各种项目和计划。但创客教育作为传入中国的教育新模 式，其本身也存在着一定的弊端和局限性。比如创客空间的 构建、创客项目所需的各种设备、器材等资源都需要大量的
经费。并且很多学校、机构设立的创客空间开放使用的时间 有限, 甚至有些只是形式, 并不开放。像有些偏远地区, 或 是资金不足的地区就无法正常开展创客教育相关活动, 这些 对于创客教育的发展有着很大的阻碍。因此虚拟实验与创客 教育的结合将会很好的解决这些问题。

\section{2. 研究现状}

虚拟实验目前主要应用于创客空间的建设。它能够为 创客爱好者提供真实实验和创客工作坊高度相似的功能 并且不受时空限制的低成本个性化学习环境。因此, 虚拟 实验在创客教育中的应用潜力是巨大的。很多专家学者也 意识到了这一点。

对于虚拟实验技术在创客教育中的应用潜力, 王同聚 [5]认为一方面, 虚拟实验能够营造出自主学习的环境, 由 传统的“以教促学”的学习方式演变为学生通过新型信息 化环境和工具来获取知识和技能的新型学习方式; 另一方 面, 虚拟实验给学生提供全新的教学工具, 激发学生学习 新知识的兴趣, 让学生在动手体验中迸发出创新的火花。 曽明星等[6]认为虚拟实验能够创设出虚拟化的实验代替 传统实验项目, 实验效果甚至优于真实实验。创客教师可 在空间进行体验式教学场景设计, 学生可进入虚拟空间实 时互动、感知和操作虚拟世界中的各种虚拟对象, 将知识 内化镶嵌在虚拟现实的社会与物理境脉中, 获得一种身临 其境的感受, 并提出自己的创新想法。徐锦霞等 [7]则认为 学习者在解决问题的过程中易受到可视化信息、模拟实验 等启发。在有些MOOCs平台如Udacity及edX中均提供虚拟 环境, 以便学习者直接进行虚拟实验, 如电子线路实验或 程序运行环境实验等。王德宇 [8]认为虚拟现实有利于学生 在与虚拟世界互动的过程中, 建构自己对教学内容的理 解, 同时整个交互过程中, 易于对操作、行为进行采集和 分析。此外, 还能够帮助学生提高探索问题、定义问题、 解决问题、交流沟通、团队合作等能力。而针对于创客教 育实际教学中的虚拟实验技术的应用, 周利平[9]认为创客 教育的虚拟实验要能围绕创客课题的创新目标, 提供充足 的创新创造素材, 这是区别创客虚拟实验与普通虚拟实验 的关键所在。并且创客虚拟实验要支持多人协作, 支持模 块化教学的特点, 这是创客教育最重要的理念之一。

综上所述，该领域分别针对虚拟实验技术和创客教育 的研究都有很多, 而关于虚拟实验在创客教育中的应用的 研究却很少, 仅有不足十篇文献。而且仅有的文献中都只 是把虚拟实验在创客教育中的应用作为一小部分提及, 并 没有对此进行专门的研究和分析。所以本文以此为出发 点, 将研究分析虚拟实验在创客教育中的具体应用, 包括 虚拟实验能够解决的创客教育遇到的问题, 以及其应用于 创客教育的特点等。

\section{3. 相关概念的界定}

\section{1. 创客教育}

中国的创客教育起步比较晚, 目前国内学术界祝智 庭、郑燕林、谢作如、杨现民等学者对创客教育的研究较 
为深刻。荆玲玲[10]在总结以上学者对创客教育的研究得 出创客教育是以培育大众创客文化和培养创客素养为导 向的一种新的教育形态，以云计算、物联网、大数据、3D 打印技术、移动互联网等信息技术的融合为基础, 以培养 创新型人才为目标, 汲取体验教育、快乐教育、做中学、 大成智慧、DIY理念、项目学习法、STEAM 教育等先进 教育理念, 并将创意、设计、实现、兴趣、团队、开放作 为整个创造过程的核心, 借助各种工具, 依托各种资源, 让学生将学习融于创造过程, 进行基于创造的学习, 培养 学生的创新思维, 实现全人发展。

\section{2. 虚拟实验}

虚拟实验从二十世纪六十年代中期发展至今, 已经经 历了由无到有、由维形的平面实验发展到四维模拟实验的 过程。目前看来, 虚拟实验随着科学技术的发展也在不断 的更新与深化。本文认为虚拟实验是指利用多媒体技术、 增强现实技术、仿真技术等信息技术在计算机上模拟进行 实物实验中的各操作环节, 实验者可以像在真实的实验环 境中一样完成各种实验项目, 所取得的实验效果等价于甚 至优于在真实环境中所取得的效果。虚拟实验建立在一个 虚拟的实验环境(平台仿真)之上, 而注重的是实验操作的 交互性和实验结果的仿真性。虚拟实验有交互性、虚拟性、 安全性、开放性的特点[11]。

\section{4. 虚拟实验在创客教育中的应用现状}

\section{1. 创客教育现存的问题与瓶颈}

创客教育自传入中国以来, 与中国的实际教育教学发 展现状相结合, 在为中国的教育注入新鲜活力的同时, 也 在兴起与快速发展中逐渐暴露出许多亟待解决的问题。比 如资源不足、师资缺乏、教学设计模式有待开发、校内创 客空间建设有待完善等[12]。而虚拟实验技术的一些特点 和优势恰好能够解决创客教育在发展中的一些问题。本文 查阅大量文献资料总结得出一下四个最主要的问题。

\subsection{1. 教师的创客教育意识浅薄}

创客教育虽然是近几年新出现的一种教育方式, 但其 背后有着丰富的教育理念, 包括杜威的“做中学”理念、项 目学习法、建构主义[13]、跨学科融合理念等。

而现在的主流教育形式还是传统教育, 创客教育还没 有达到极高的认可度, 并且目前从事创客教育的老师也都 是传统教育出身, 在进行创客教学课程设计上还是沿用传 统教育的教学模式、评价方式、测试和思路, 即大量的传 授相关课程知识, 让学生被动的接受知识, 以考试或者测 试的形式检验学生的学习效果, 而对于学生是否能够灵活 运用相关知识进行实践并没有作为教学重点, 这与创客教 育强调的“做中学”严重相悖。而各个学校的老师本身对于 创客教育的理解不够深入, 也缺乏相关的创客教育实践, 因此没有主动积极参与创客教育的意识与行动, 这大大阻 碍了创客教育的普及与发展。

\subsection{2. 创客教育沦为中小学的“面子工程”}

目前创客教育极其火爆。中国自2014年起陆续出现很 多创客公司, 各大高校也纷纷启动了一系列与创客教育相 关的项目或计划, 中小学也将创客教育引进校园, 建设创 客空间, 参加各种创客创新大赛等等。开展创客教育似乎 成为一种潮流, 但真正实现常态化的学校并不多, 甚至在 一些所谓的创客教育示范校里, 也只是新建了几间新型教 室, 费尽心思打磨几节观摩课, 等活动结束后就把创客教 育抛于脑后[14]。相关的场所、设备、资源也不会让学生 使用。有些学校甚至把科技大赛获奖多少当做创客教育的 成果, 花大量的人力物力财力, 只是为了推出个别特长生 为学校争得荣誉。创客教育似乎沦为各大学校追逐政绩的 工具, 如果任由这种现象发展下去, 那么创客教育无法普 及, 其本身具有的价值也就不会改善当前的教育现状, 创 客教育终将会消失。

\subsection{3. 创客空间封闭落伍, 开放性有限}

中国的学校关于创客教育的实施, 虽然有一些知名学 校会投入大量资金建立专业化程度比较高的创客空间 [15], 但其他大多数是在已有的图书馆、实验室、科研活 动中心等场所和设备的基础上进行改造。因此学校所能提 供给学生用于创客教育的资源有限, 很多设备也比较老 旧, 器材落后, 校方也没有足够的资金来配置配套的物资 资源。这会导致学生的创意、想法因为资源不足而无法实 践检验, 长期下去会消磨学生的创新热情, 降低学生的创 造意愿[10], 不利于创客教育的开展。

此外, 部分学校的创客空间并不是长期开放, 而是有 固定的开放时间, 甚至只是为了应付检查而暂时开放一 次。这可能会与学生正常的上课时间冲突, 而空闲时间又 无法进入创客空间学习和实践。大部分学校在寒暑假是不 会对外开放的, 这会使师生、生生之间分享创意、合作学 习的机会大大减少。其次, 很多学校只是对本校的师生开 放, 对校外人士拒绝开放, 把创客空间当做是校内资源, 这大大阻碍了学校师生与社会上的创客群体交流, 也违背 了创客空间的共享理念。

\subsection{4. 创客教学过程中忽略学科间的联系}

多学科融合是创客教育的支撑理念之一。在创客教学 项目中, 一般不会只涉及某一学科的知识, 而是多个学科 的复杂问题。学习者需要在一个项目实践中综合运用多种 学科知识, 解决过程中遇到的问题, 这就需要创客教学团 队的老师也要懂得各个学科的知识。而现在很多学校把创 客教育等同于信息技术，甚至有些学校直接让信息技术老 师负责创客课程的教学, 而其他学科的老师更是认为创客 教育与他们无关。大多数教师在授课的过程中也只是传授 单一的学科知识, 甚至根本没有跨学科相融合的意识, 只 是浅显的把创客教育归于某一种前沿的技术应用, 新潮的 硬件开发。这将不利于创客教育的发展。

\section{2. 虚拟实验在创客教育中的应用特点}

从虚拟实验技术近年来在教育教学上的广泛应用可 以看出, 虚拟实验技术十分符合新时代对人才培养的要 
求。而虚拟实验若与创客教育相结合, 则会很好的解决上 述创客教育在中国教育实际发展中暴露出的问题。

\subsection{1. 互动性, 以学生为中心}

在虚拟实验平台上, 学习者可用鼠标拖动、点击实验 器材进行实验探究, 实现人机交互, 可以观察到交互之后 的结果一一实验数据与实验现象 [14]。除此之外, 在虚拟 实验平台上, 所有的用户均可互动交流, 包括教师、学生 以及社会上的创客。这种人机互动和人人互动正是虚拟实 验应用于创客教育的主要特点之一。

而创客教育是以学习者为中心的教育模式, 在虚拟实 验平台上, 无论是学生还是教师都在互相交流、学习。学 生不再只是“听众”, 只能被动地接受知识, 而是学习的“中 心”[8]; 教师不再只是传授知识, 也会在项目中学到新知 识, 这不仅有利于学生综合能力的提升, 也有利于教师创 客教育意识的树立和加强。

\subsection{2. 共享性}

虚拟实验平台上的实验器材、专业设备等实验所需资 源可供多个学习者同时使用, 不受时空限制, 不仅如此虚 拟实验平台可提供给学习者大量的相关学科的资源, 供学 习者在项目的推进过程中不断的学习, 也不会在找资料上 面花费过多的时间。虚拟实验的“共享性”可实现学校的创 客教育的常态化, 使所有学生均可自如的参与到创客过程 中。

\subsection{3. 开放性}

虚拟实验在创客教育中应用的主要特点之一就是具 有开放性, 可以让学习者按照自己的想法、创意进行实验 的设计与实现探究, 不受时空、地点、年龄等条件的限制。 而且现在全国各地支持创客教育的资金、资源、师资力量 条件不尽相同，而虚拟实验就可以打破这些条件的限制实 现全国甚至全球范围内创客爱好者的交流互动。

\subsection{4. 跨学科知识整合}

在创客教育的实践教学中, 教师大多数只以某一学科 为主, 其他学科知识甚至不会涉及。而虚拟实验将会在平 台上提供给学习者多学科的学习资源, 并且在实验的过程 中也会涉及到多学科知识的综合运用, 这将让学生在项目 的完成过程中综合学习各学科的知识并掌握。虚拟实验的 跨学科知识整合的特点正契合了创客教育中的多学科综 合的教育理念, 有利于学生的综合素质的提高。

\section{3. 虚拟实验在创客教育中的应用设计原则}

\subsection{1. 虚实结合、互相补充、能实不虚}

虚拟实验在创客教育中的应用应本着“虚实结合、相 互补充、能实不虚”的原则, 实现真实实验不具备或难以 完成的教学功能。在涉及高危或极端的环境, 不可及或不 可逆的操作, 高成本、高消耗、大型或综合训练等情况时, 提供可靠、安全和经济的实验项目 [16]。

\subsection{2. 支持“按需学习”}

创客教育作为一种新的教育方式, 其面对的人群是社 会上的所有学习群体, 不单单是在校学生。因此, 创客教 育中的虚拟实验必须要满足所有人的学习需求, 为不同类 型的学习者提供适时、适地、适当的学习服务, 实现无缝 化按需学习[15]。

\subsection{3. 创新性}

创客教育旨在创新, 因此创客虚拟实验也应具备创新 性的应用原则, 这是区别于普通虚拟实验的关键。创客教 育中的虚拟实验应给参与者提供尽可能多的创新素材, 满 足他们在实践操作过程中的创新需求, 实现自己的创意想 象, 提升自身的创新创造能力。

\subsection{4. 支持多人协作}

创客教育的主要目的之一是培养学习者的团队协作 能力, 因此虚拟实验在创客教育中应采用多人协作的模 式, 多人合作完成实验, 在解决问题的过程中与成员的沟 通交流中提高自己。这也是创客教育区别于传统教育的人 才培养目标的关键所在。

\section{4. 虚拟实验在创客教育中的应用教学模式}

创客教育注重培养学生的综合实践能力、创新能力。 因此, 创客教育的教学模式更加注重“做中学”的教学理 念。而虚拟实验的出现, 则会消除创客教育教学中的客观 限制因素。因此, 本文将创客教育与虚拟实验相结合, 总 结得出虚拟实验应用于创客教育的应用教学模式 (见图 1）。在该教学模式中, 除了“创设情境, 确认主题”是在 线下老师与学生面对面进行之外, 其余的教学环节均可在 虚拟实验平台上进行, 也可线上线下同时进行。

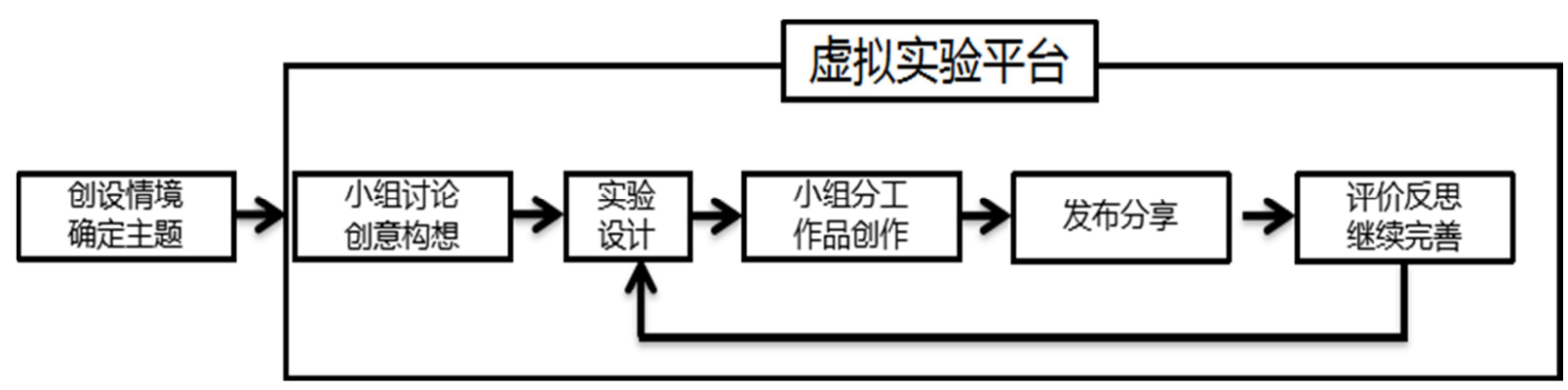

图1 虚拟实验在创客教育中的应用教学模式。 
（1）创设情境，确认主题：教师利用网络空间上的 视频、动画、案例等交互性的媒体资源创设问题情境 [17], 并确认项目相关主题, 提供给学生相关的学习资源。

（2）小组讨论, 创意构想: 学生分小组进行问题讨 论, 构思创意, 可在虚拟实验平台上进行讨论, 也可线下 进行。

（3）实验设计: 小组内根据本组的课题及创新点进 行项目框架的搭建, 并完成相关实验设计。

（4）小组分工，作品创作：小组成员在虚拟实验平 台上分工进行作品的创作。

(5) 发布分享: 小组将本组作品上传至虚拟实验平 台的分享讨论区, 并进行简要介绍, 老师及学生进行自评 与互评。

（6）评价反思，改进完善：结合平台上老师的意见 以及同学之间的互评结果汇总反馈, 对自己小组的作品进 行改进和完善, 形成改进方案并返回第 (3) 步分工实施。

\section{5. 虚拟实验在创客教育中的应用优势}

\subsection{1. 经济、方便}

虚拟实验不仅能够提供给学习者创客实体空间的功 能, 而且在虚拟实验平台上还能够实现所有人员的无阻碍 交流, 不需要购买硬件材料、设备, 也不需要特定场所供 师生交流实践, 师生可不受时空限制, 按照自己的时间安 排在虚拟实验平台进行创客实践。这在一定程度上节约了 创客教育的运行成本, 有利于创客教育更快速度的普及发 展。

\subsection{2. 学习效果优于实体教学}

在真实实验中, 学生只能按照老师讲解的步骤一步一 步的完成才能达到实验的效果, 并且在实验过程中每一个 步骤呈现的效果都是一成不变的。而虚拟实验在进行过程 中, 学生可按照自己的想法改变实验过程中的任意一个条 件来观察最终实验呈现的结果。而且虚拟实验是利用先进 的计算机技术、虚拟现实技术等来创建虚拟实验环境, 是 对现实世界的虚拟, 不仅要模拟现实世界真实存在的现 象, 还要虚拟现实世界难以达到的理想状态 [14]。因此, 在创客教育中采用虚拟实验会使学习者的学习体验达到 最佳, 学习效果也比实体教学更显著。

\subsection{3. 安全性能高}

在创客教育教学实验过程中, 最为关注的就是安全问 题。而创客教育强调跨学科融合, 学生在解决一个问题的 过程中必然会接触到不同学科的知识, 有些知识在真实情 况下由于实验的安全隐患导致学生只能通过图文或者视 频来了解。而虚拟实验在创客教学过程中就可以排除掉这 些安全隐患, 让学生亲身学习实践。虚拟实验的研发提升 了学生学习的兴趣, 同时改善了实验教学中存在的问题 [18]。

\section{5. 虚拟实验在创客教育中的应用实例}

虚拟实验技术正以不可估量的速度发展, 影响着人们 的生活、工作、学习。近年来, 3D打印、移动学习、虚拟 实验等技术愈来愈多的应用于教育教学实践中。业界把 2017年称为“VR创客教育”元年, 虚拟现实如何与创客教 育融合也成为了 2018 新型信息化教育的重点发展方向 [19]。

中国也有一些团队注意到虚拟实验技术可以解决创 客教育在发展中出现的问题, 并付诸于实践, 比如云幻教 育科技股份有限公司的团队的创新教学产品一 $\mathrm{AR}$ 物理仿 真实验室 [20]。云幻科教 AR物理仿真实验室综合应用 $\mathrm{AI}$ 和AR技术, 通过 $3 \mathrm{D}$ 建模虚拟再现真实的实验场景, 借助 先进的体感交互设备Kinect识别操作者的手势, 与画面中 虚拟的实验器材进行互动[20], 这使得学生对一些实体高 危险性实验的操作和完成成为可能, 从而有利于学生对于 知识的理解和掌握。同时, AR物理仿真实验室解决方案 整合常规教室与实验室, 搭建校园创客空间与教学空间, 为学生探索与实践提供优质的实验器材, 虚拟实验产生的 数据与真实实验器材产生的数据相同[20]。学生们将头脑 中的想法利用虚拟实验探究形成系统的、可执行的方案, 使得学生可以将自己的创意想法付诸实践, 增强学生的创 新意识, 提供动手能力与创新能力。不仅如此, 学生在实 践探究之中发现问题、解决问题, 从而从中习得广泛的经 验、技能、知识等。AR物理仿真实验室正是通过虚拟实 验相关技术应用于创客教育, 改变学生的学习方式, 基于 学生的学习兴趣, 培养学生的创新能力与实践能力, 推进 创客教育发展。

而在高校的课程中也引入虚拟实验技术, 让学生在完 成项目、任务的过程中更好的提升自身的能力。比如湖北 大学2015 级计算机与信息工程学院电子信息工程、通信 工程和通信工程专业三个班级的学生参与完成电子线路 PSpice仿真课程 [21]。该系列课程中包括利用虚拟仿真 PSpice软件完成高频微弱声音监测实验和利用虚拟仿真 Protel软件完成图像采集与处理实验。在这两个利用虚拟 实验的具体教学实践中, 学生对于元件选取、参数设置、 元件连接等实验中需要考虑的问题按照自己的理解进行 尝试, 并对产生的功能效果进行比对, 在反复的修改、思 考、实践中对课程内容有了更加深刻的理解[21]。而在图 像采集与处理的过程中, 小组成员分工利用虚拟仿真软件 完成自己负责的模块, 最终再合成在一起。在实践过程中 利用虚拟实验软件, 每个成员都在互相交流协作, 共同完 成实验项目, 同时也很好的掌握了相关的理论知识。

\section{6. 虚拟实验应用于创客教育的价值}

\section{1. 极大的满足学习者的创新需求}

创客教育是创客运动与教育的结合, 旨在用项目的方 式来让学生进行创新实践。而由于实体的创客空间、所用 到的设备、材料在实践过程中会存在很多安全性的问题及 
一些客观限制, 因此学生的很多创意、想法在真实的实践 过程中无法得到实现, 这将会逐渐降低学生的积极性。而 虚拟实验完全解决了这一问题, 在虚拟实验空间中, 学生 无需考虑安全性等客观限制, 只需要按照自己的想法进行 实验探究, 在这个过程中不断的摸索, 解决问题, 实现自 己的创新。因此, 虚拟实验能极大的满足学习者的创造热 情和创新想法, 从而能够提升他们的创新能力和问题解决 能力。

\section{2. 扩宽了创客教育的普及范围}

中国各地区之间教育资源分配不均, 经济发达地区无 论是软硬件配置、创客空间的建设专业化程度都非常高, 创客教育的研发团队也有很多; 而经济落后或者偏远地 区, 学生的基础教育都无法保证, 对于创客教育更是无力 支持。而虚拟实验应用于创客教育, 将会极大的解决这一 问题，有利于缓解创客教育地区发展不平衡的现象，扩大 优质资源的分享范围, 让全国各地的创客们平等的享受所 有优质的教育资源, 都有机会实现自己的创意、想法。这 对于创客教育的普及与发展有着至关重要的意义。

\section{3. 实现寓教于乐、寓学于乐的结合}

创客教育倡导的是以学习者为主导的自主学习模式, 教师只是起着指导、辅助的作用。而在虚拟学习空间里, 教学体现为寓教于乐和寓学于乐的相互结合, 让学生体验 身临其境的学习感受, 为课堂教学和自我学习提供了支持 [22]。虚拟实验利用了多媒体技术、增强现实技术、仿真 技术等信息技术在计算机上模拟进行实物实验中的各操 作环节, 这极大地增强了学习过程的趣味性, 给学生的学 习带来强烈的亲身体验感受, 学生会更加积极、主动的投 入学习。尤其是在中小学的创客教育教学过程中, 效果更 为明显。

\section{7. 结论}

本文通过阅读虚拟实验技术及创客教育相关的文献， 对虚拟实验在创客教育中的应用进行探讨, 从其应用的特 点、原则、相应的教学模式、优势以及应用意义进行阐述。 随着时代发展, 传统教育所存在的弊端日益显现, 创客教 育的出现正是人们寻求更加符合国家发展需求的新型教 育方式的结果。让学习者运用先进信息技术, 结合自己所 学的知识, 把自己的创意实现出来, 提高自身的创新能力 和综合素质以及与他人写作的能力, 这是创客教育的初 衷。而虚拟实验的助力, 将会解决创客教育在发展过程中 所遇到的问题。

目前的创客教育大多数还是实体的教学环境, 虽然已 有很多的创客空间采用 020 的模式, 但是以线下实体教学 为主, 线上虚拟创客空间为主。虚拟实验在目前的创客教 育实际的实践应用中只是起到很小的一部分功能, 即学生 用来进行课程中的实验练习, 其目的也仅仅是让学生有着 身临其境的体验感受。这或许是与当前的虚拟实验的发展 情况有关。
相信在不久的将来，虚拟实验将会实现创客教育的更 多功能的替代, 学生不只是只能在虚拟实验平台上做已知 的实验任务, 而是真正能借助虚拟实验, 不用考虑安全性、 经济性等一切限制因素, 按照自己的想法进行探索, 实现 自己的想法, 解决问题, 进而完成项目。虚拟实验技术将 会更加注重于当代学生对科学知识的学习、对创新意识的 认知、对自主学习的培养、对新事物的探索的能力。虚拟 实验技术在创客教育中的应用在当今社会网络世界中有 着广阔的发展前景, 对于世界经济的快速发展有着积极的 促进作用。

\section{致谢}

感谢北京邮电大学教育技术研究所文福安教授在文 章写作过程中给予的指导和帮助。

\section{参考文献}

[1] 傅骞,郑娅峰.创客教育区域推进策略研究 [J].中国电化教 育,2018(05):61-68。

[2]国家创新驱动发展战略纲要 [ $\mathrm{EB} / \mathrm{OL}$ ] http://www.xinhuanet.com/politics/2016-05/19/c_1118898033 .htm

[3] 胡瑞文. 《国家中长期教育改革和发展规划纲要》主要精神 解读与热点、难点探析 $[\mathrm{J}]$. 中国高等教育评 估,2010,22(02):3-10。

[4] 王伟民.改革传统教育弊端 推行创新教育理念 [J].高等教育 研究学报,2002(04):73-74。

[5] 王同聚.虚拟和增强现实(VR/AR)技术在教学中的应用与前 景展望[J].数字教育,2017,3(01):1-10。

[6] 曾明星,宁小浩,周清平,徐洪智,粟娟.面向大学生创客的网 络学习空间构建 [J].中国电化教育,2016(11):30-38。

[7] 徐锦霞,钱小龙. 基于体验学习理论的MOOCs教学系统构建 研究[J].外国教育研究,2014,41(09):74-84。

[8]王德宇,宋述强,陈震.增强现实技术在高校创客教育中的应 用[J].中国电化教育,2016(10):112-115。

[9] 周利平.基于远程学习平台的线上创客空间模型设计 [J].广 东开放大学学报,2018,27(01):1-6。

[10] 荆玲玲, 刘庚. 创客教育的内涵要素、问题反思及发展路径 [J]. 教学与管理,2018(03):1-4。

[11] 李丽芳. 虚拟实验在初中物理实验教学中的应用研究[D]. 南昌大学, 2017 。

[12] 徐丽. 走进 “创客”式学习的新时代 [J]. 中 小学管 理,2018(08):50-51。

[13] 白敏,高杰.创客教育实践过程及问题研究[J].中国教育信息 化,2016(14):13-16。 
[14] 李丽芳. 虚拟实验在初中物理实验教学中的应用研究 $[D]$. 南昌大学,2017。

[15] 祝智庭, 孙妍妍. 无缝学习——数字时代学习的新常态 [J]. 开 放教育研究,2015,21(01):11-16。

[16] 教育部办公厅关于开展2015年国家级虚拟仿真实验教学中 心 建 设 工 作 的通知 http://www.moe.gov.cn/srcsite/A08/s7945/s7946/201506/t201 50618_190671.html

[17] 杨晓粀,谢幼如,钟如光.网络空间支持的中小学创客教学模 式研究[J].电化教育研究,2017,38(01):101-107。

[18] 杨蕾. 基于虚拟实验学生科学探究与创新意识的培养 $[D]$. 哈尔滨师范大学,2017。
[19] 虚拟现实如何在创客教育应用这里有个答卷 http://www.caigou.com.cn/news/2018051146.shtml

[20] $\mathrm{AR}$ 物 理 仿 真实验促进创客发展 http://www.nbjyj.com/hdjl/zwzx/20180208/11994.html

[21] 盘亚楠,谌雨章,郭显玮.虚拟仿真与创客教育的融合与实践 —电子线路仿真类课程教学改革研究 $[\mathrm{J}]$. 科技视 界,2018(07):6-10。

[22] 杜星月. 基于混合式学习的学习空间构建及其应用研究[D]. 山西师范大学,2017。 